\title{
Thromboembolic Events in Multiple Myeloma Patients- Incidence in 235 Patients
}

\begin{abstract}
Alves $C^{1 *}$, Ramalheira $\mathrm{S}^{2}$, Tavares $\mathbf{M}^{2}$, Simas $\mathrm{A}^{3}$, Rosales M', Milheiro MJ ${ }^{1}$, Domingues $\mathbf{N}^{2}$, Chacim $\mathrm{S}^{2}$, Santos $\mathrm{LL}^{1}$ and Mariz J M ${ }^{2}$

${ }^{1}$ Department of Imuno-Hemotherapy, Instituto Português de Oncologia do Porto Francisco Gentil, Portugal ${ }^{2}$ Department of Hemathology, Instituto Português de Oncologia do Porto Francisco Gentil, Portugal ${ }^{3}$ Department of Medical Oncology, Instituto Português de Oncologia do Porto Francisco Gentil, Portugal

*Corresponding author: Alves C, Department of Imuno-Hemotherapy, Instituto Português de Oncologia do Porto Francisco Gentil, EPE, Rua Dr. António Bernardino de Almeida, 4200 Porto, Portugal
\end{abstract}

Received: January 05, 2017; Accepted: February 13, 2017; Published: February 16, 2017

\begin{abstract}
Background: Cancer patients are more likely to develop venous thrombotic events (VTE). The incidence is higher in patients with hematologic malignancies and in patients with multiple myeloma (MM). The incidence of VTE in cancer patients with MM varies from 3 to $10 \%$, which is associated with a poor prognosis and a decreased quality of life.
\end{abstract}

Patients and Methods: A single centre retrospective analysis of patients with MM, diagnosed between 2009 and 2014, was made with the intention to identify the VTE which had occurred.

Results: We identified 235 patients with MM and registered 19 VTE-13 deep venous thrombosis (DVT), 4 pulmonary embolisms (PE), 1 superficial venous thrombosis (SVT) and 1 stroke. The incidence of VTE was $8 \%$. No significant individual risk factors were found. Nine patients were not on prophylactic therapeutics, 6 were doing ASA, 3 were under low molecular weight heparin $(\mathrm{LMWH})$ and 1 was doing acenocoumarol. The mean time between diagnosis and VTE was 8.5 months.

Conclusion: The 8\% VTE incidence found for this cohort highlights the need for VTE prophylaxis in these patients with MM.

Keywords: Incidence; Multiple myeloma; Prophylaxis; Risk factors; Thrombosis

\section{Abbreviations}

ASA: Acetylsalicylic Acid; DVT: Deep Venous Thrombosis; LMWH: Low Molecular Weight Heparin; MM: Multiple Myeloma; MPT: Melphalan, Prednisone, Thalidomide; PE: Pulmonary Embolism; SVT: Superficial Venous; TaCyDex: Thalidomide Cyclophosphamide Dexamethasone; TD: Thalidomide Dexamethasone; VMP: Bortezomib Melphalan Prednisone; VTD-PACE: Bortezomib Thalidomide Dexamethasone Cisplatin Cyclophosphamide Etoposide Doxorrubicin; vTD: Bortezomib Thalidomide Dexamethasone; VTE: Venous Thromboembolic Events

\section{Introduction}

Cancer is an independent and major risk factor for VTE and is described as the second leading cause of death in these patients [1]. The association between cancer and thrombosis is well established and known since 1865 when Armand Trousseau first described it [1-3]. Cancer associated thrombosis affects cancer patients lives significantly and is an increasing factor of morbidity and mortality [2-4]. VTE is defined as DVT or PE, and reported in up to $20 \%$ of cancer patients, $78 \%$ of which occur in outpatient settings $[1,5,6]$. In hospitalized cancer patients, several studies have noticed an incidence of VTE of $0.6 \%$ to $7.8 \%$ [5]. There are some risk factors that can enhance the incidence of VTE in cancer patients. The highest rates of VTE events are associated with the stage and type of cancer, presence of metastatic disease, use of antineoplastic therapy, as well as individual risk factors. Pancreas, brain, ovary, uterus, kidney, stomach, colon, rectum and lung cancers have the highest incidence.
However, hematologic malignancies, in particular, leukaemia, Hodgkin disease, non Hodgkin lymphoma and MM, have high rates of VTE, ranging from $4.2 \%$ to $5 \%$. The risk of VTE varies over time after the diagnosis of cancer and is higher early after the diagnosis [5,7]. Furthermore, VTE has serious clinical consequences such as an increased risk of recurrence, post-thrombotic syndrome, pulmonary hypertension, bleeding complications related to treatment and overuse of health resources $[1,5]$. As can be understood, the risk of VTE in cancer patients and the consequences of DVT or PE in this population, accentuates the need for healthcare professionals to carefully assess patient-, cancer-, and treatment-related factors when treating patients with medical oncology settings [5].

$\mathrm{MM}$ is an independent risk factor for VTE and, ever since the introduction of the immunomodulatory drugs, thalidomide and/ or lenalidomide, the risk for VTE has increased, particularly in new diagnosed patients and when combined with dexamethasone or chemotherapy. Higher doses of corticotherapy, erythropoietin, presence of central venous catheter, infections, immobilization due to skeletal pain and hospitalization are factors that also contribute to the increase of VTE in MM [8-11].

The goal of this study is to evaluate and characterize the VTE in MM patients in our institution.

\section{Materials and Methods}

This study is a descriptive and retrospective analysis of the medical records of all consecutive patients with newly diagnosed MM and VTE, between January of 2009 and December of 2014, at 
Table 1: Risk-assessment model for the management of VTE in MM patients, Palumbo A, et al. [12].

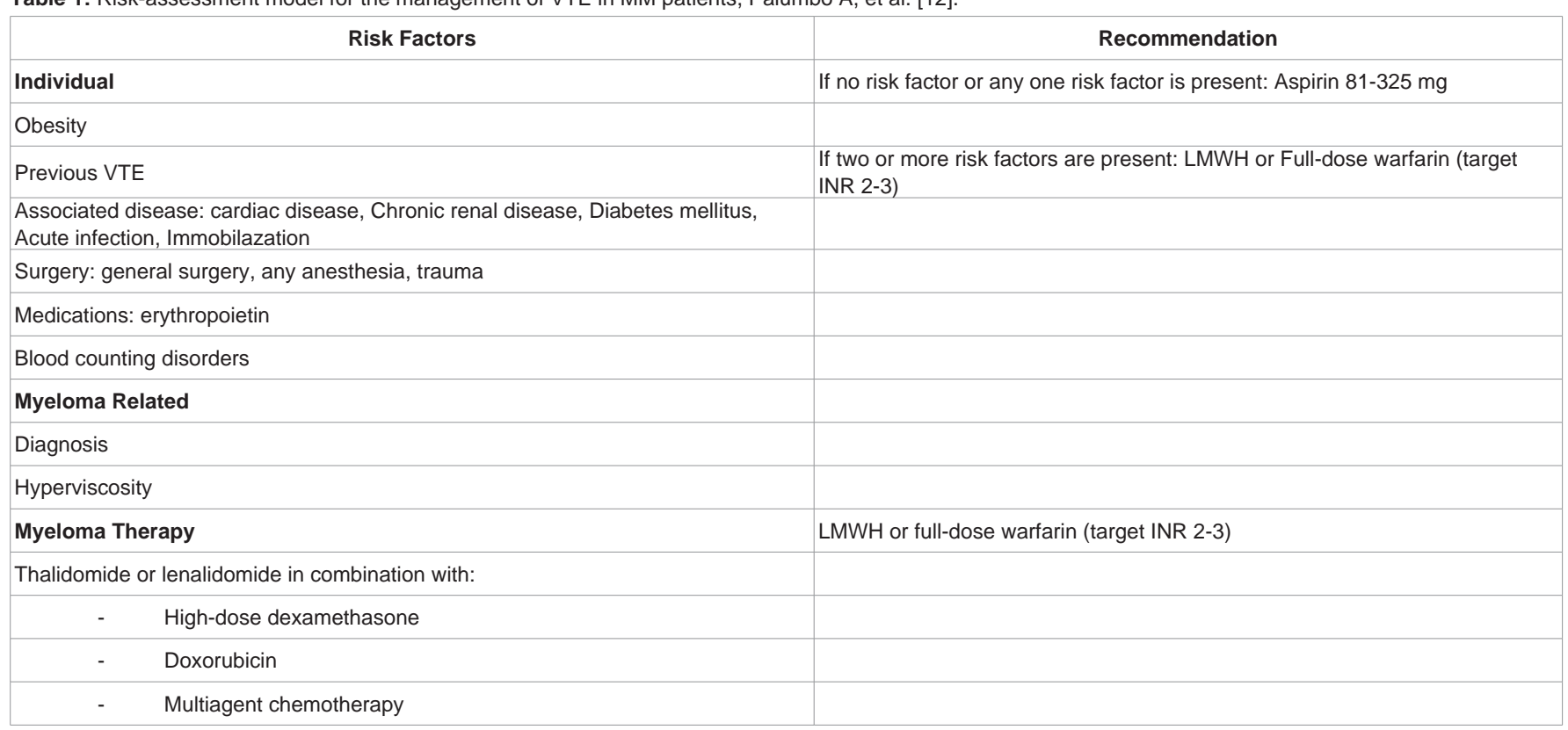

Instituto Português de Oncologia do Porto (IPO Porto). IPO do Porto, is a fourth level hospital located in northern Portugal and specialized in oncology patients. It receives a mean of 10,000 new patients/year from all northern regions of the country and 500 of them are hematologic patients.

We characterized our study population for sex distribution, median age, date of diagnosis and the treatment regimen applied. In our institution, at the time of the diagnosis of MM, all the patients were assessed for risk factors, individual and related to MM, with Palumbo, et al. [11] risk assessment model (Table 1) in order to apply the adequate prophylactic therapeutic measures, we analysed the patients with MM and VTE concerning the prophylactic therapeutic used. This analyse was not applied to the remaining MM patients because it was out of the field of this study.

Regarding the VTE, we estimated the cumulative incidence of VTE considering the competing risk of death and we characterized thromboembolic events according to the mean time between the diagnosis of myeloma and the VTE; the type of event and diagnosis method, the antithrombotic therapeutic instituted and the outcome of the patients after the VTE. All VTE were ascertained by the information provided in the eletronic clinical files of each patient, which has crosslink information with all national health system Instituions, we consider that there were no missed VTE events. Finally, we used a survival analyses to conclude if there was any statistical difference between the survival of MM patients with and without VTE.

\section{Results}

We identified 235 patients with MM in our hospital and 19 of these had a thromboembolic event (11 males; median age: 69 years old; range 51-80 years old), which correspond a cumulative incidence of $8.1 \%$ (Figure 1). In this group 14 were ambulatory patients and 5 were hospitalized. At the time of the thrombotic event 9 patients were receiving treatment: 5 with vtD regimen (Bortezomib, Thalidomide

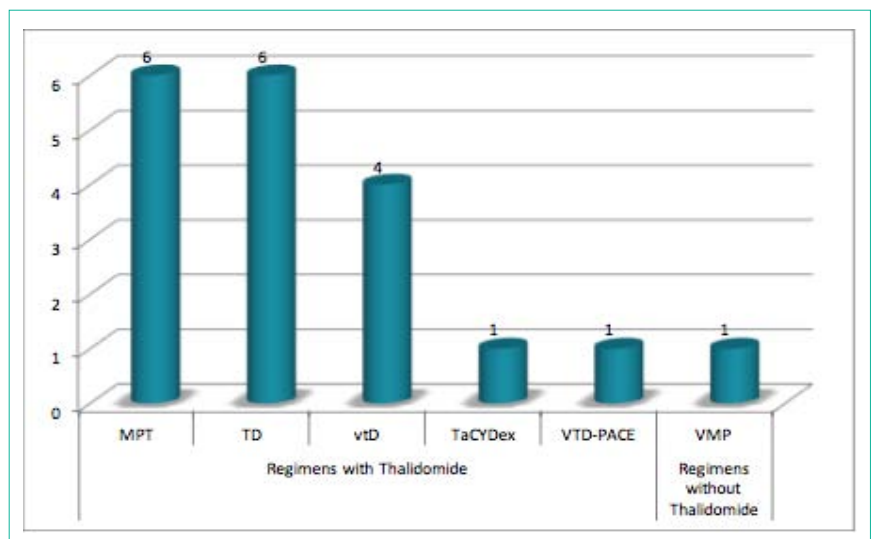

Figure 1: Therapeutic regimen applied at the time of VTE diagnosis.

and Dexamethasone), 2 with Lenalidomide and Dexamethasone and 2 with Bortezomib and Dexamethasone, which correspond in 5 of them to the first line treatment and the 4 remaining to the second line treatment. The treatment regimen used to control the myeloma in these patients is represented in Figure 2

In relation to the use of prophylactic measures, 6 out of the 19 were under ASA, 3 were using LMWH and 1 were under acenocoumarol. In what concerns the 9 remaining patients, they were not taking any prophylactic measures according to different causes. In 3 of them the diagnosis of VTE and MM was coincident and previous to any antineoplastic treatment; 3 didn't have any prophylactic regimen at all; 2 were diagnosed outside of our institution and we couldn't evaluate the use of prophylactic measures and 1 had the diagnosis of VTE before MM. Regarding the VTE, the mean time between the diagnosis and the VTE was 8.5 months. The distribution for type of event is represented in Figure 3. The main diagnosis method was doppler ultrasound (10 patients), followed by computerized tomography scan (5 patients) and 1 simple ultrasound, in the 3 remaining there was no register. All of the VTE were first events and there was no any 


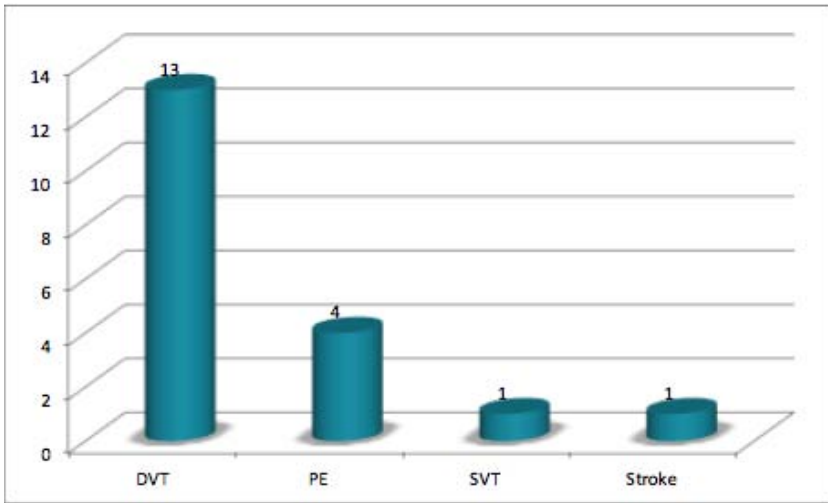

Figure 2: Distribution for type of VTE in the study population.

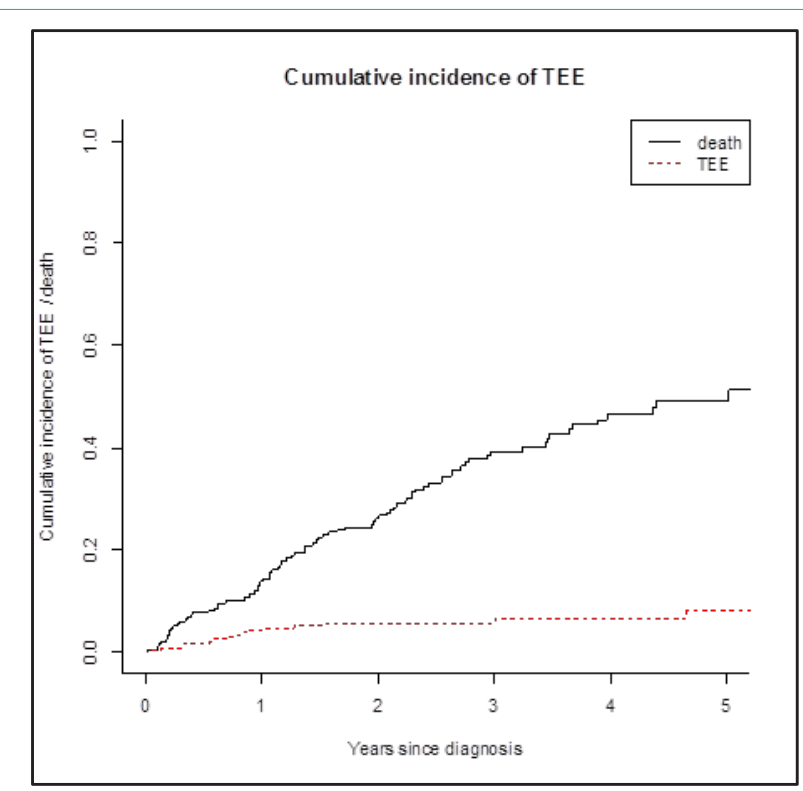

Figure 3: Cumulative Incidence of Thrombotic Events.

recurrent events. At the time of this study all of the 19 patients were under anticoagulant treatment (14 with warfarin, 3 with LMWH and 2 with acenocoumarol) but all of them started with a 3 to 6 months period of LMWH in a weight adjusted dose before taking the oral anticoagulants.

The survival analysis is represented in Figure 4 and there was not a significant statistically difference $(p=0.630)$ between patients with MM and VTE and patients with MM without VTE.

\section{Discussion}

According to the literature, cancer patients have an increased risk of thrombosis by 4 to 5 folds, rather than the general population and the risk is considerably higher in patients with hematologic cancer. VTE is multifactorial and is often a consequence of a combination of risk factors $[10,11]$.

Thromboembolic risk factors can be categorized into individual risk factors, such as older age, comorbid conditions, central venous catheter or pacemaker, previous history of thrombosis, pregnancy

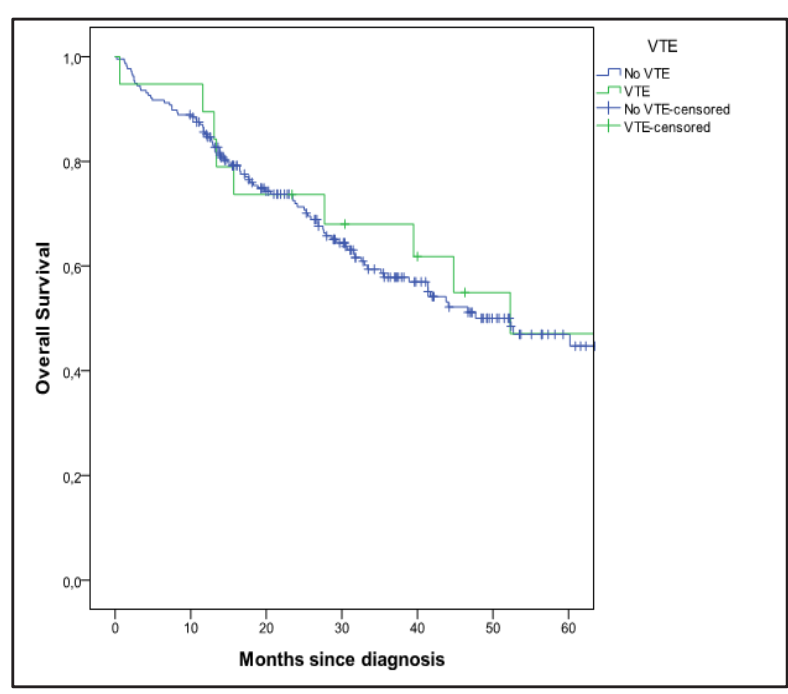

Figure 4: Survival analysis.

or puerperium, use of drugs, heritable prothrombotic mutations and inherited thrombophilic abnormalities, and disease-related risk factors which are: recent surgical procedures, prolonged immobilization, chronic clinical conditions, primary site of the cancer, initial 3-6 months after diagnosis, use of cytotoxic drugs, antiangiogenic therapy such as thalidomide, lenalidomide or bevacizumab and erythropoiesis-stimulating agents. The simultaneous presence of more than one risk factor increases the risk of VTE $[4,10,11]$.

$\mathrm{MM}$, in particular, is associated with an increased thromboembolic risk but the exact mechanisms implicated in this process are still unclear. The disease risk factors can derive from the presence of the monoclonal component and from the procoagulant activity of inflammatory mediators. The $\mathrm{M}$-component can be directed against different hemostatic pathways, leading either to bleeding, which is rare, or thrombotic tendency $[10,12]$. Also the treatment regimens, such as thalidomide and lenalidomide, can contribute to the thrombogenic tendency. Thalidomide, mostly when is used in association with dexamethasone, increases significantly the incidence of VTE $[8,10$ 12]. Similarly, lenalidomide, which is an immonumodulatory drug that is structurally similar to thalidomide, yet functionally distinct, has a thrombogenic activity when combined with dexamethasone $[8,11,12]$. In our study population, we identified a majority of risk factors associated with the MM and only a minority had individual risk factors. When we evaluate the risk factors of the 19 patients based on the Palumbo, et al. [11], we analyse the appropriateness of the 10 patients that were receiving prophylactic therapeutic measures (9 patients weren't on any prophylactic measures as stated in the results). The six patients on aspirin only had one individual risk factor, but 3 of them should be on LMWH or oral anti-coagulation because of the associated thalidomide+dexamethasone treatment. Considering the 4 patients that were taking LMWH/acenocoumarol we may consider that only 2 could be on aspirin because they had only 1 risk factor and the other 2 were appropriately anti-coagulated. Despite the prescription of VTE prophylaxis according Palumbo's guidelines, we didn't collect any data regarding the adherence of the 235 patients to this prescription. 
In the literature, the incidence of VTE in MM patients varies from $3 \%$ to $10 \%$, increasing with the use of thalidomide or lenalidomide and with the association of these drugs to corticosteroids [4,6,8-12]. The $8 \%$ incidence found in our study is in accordance with the data published.

As we can see from the data presented, in MM patients it is important to define a prophylactic strategy to prevent VTE. The choice of thromboprophylaxis needs to be adjusted to the risk stratification of the patient. The main goal should be to use the safest and least cumbersome form of thromboprophylaxis that may reduce the risk of VTE to at least below 10\% [8]. According to the guidelines, MM patients who do not have risk of bleeding and are treated with thalidomide or lenalidomide in combination with dexamethasone or chemotherapy, should receive thromboprophylaxis. In thalidomide or lenalidomide treated patients, LMWH or a full dose warfarin is recommended if the patient has two or more individual risk factors. If there are any individual risk factors or only one risk factor, the prophylaxis should be performed with ASA $[8,10,11]$. The decision about the prophylactic treatment of our 10 patients was based on the risk stratification defined at the first visit at our institution.

\section{Conclusions}

This study enhances the importance of VTE in MM patients. As we can conclude from the data presented, VTE has a high incidence which outlines the need for applying accurate prophylactic therapeutic regimens, in order to prevent VTE and improve patients' quality of life and overall survival.

\section{Acknowledgement}

The authors would like to thank to Luís Antunes, of the statistical department of our Institution, for his contribution to the statistical analyses of our data.

\section{References}

1. Farge D, Debourdeau P, Beckers M, Baglin C, Bauersachs RM, Brenner B, et al. International clinical practice guidelines for the treatment and prophylaxis of venous thromboembolism in patients with cancer. $\mathrm{J}$ Thromb Haemost. 2013;11:56-70.

2. Streiff MB. Association Between Cancer Types, Cancer Treatments, and Venous Thromboembolism in Medical Oncology Patients. Clin Advan Hematol Oncol. 2013; 11: 349-357.

3. Donati MB. Thrombosis and cancer: Trousseau syndrome revisited. Best Pract Res Clin Haematol. 2009; 22: 3-8.

4. Khorana AA. Cancer-associated thrombosis: updates and controversies. Hematology Am Soc Hematol Educ Program. 2012; 626-630.

5. Lee AY, Peterson EA. Treatment of cancer-associated thrombosis. Blood 2013; 122: 2310-2317.

6. Mandalà $M$, Falanga $A$, Roila $F$. Management of venous tromboembolism (VTE) in cancer patients: ESMO Clinical Practice Guidelines. Ann Oncol. 2011; 22: vi85-vi92.

7. Conolly GC, Francis CW. Cancer-associated thrombosis. Hematology Am Soc Hematol Educ Program. 2013; 684-691.

8. Khorana AA, Kuderer NM, Culakova E, Lyman GH, Francis CW. Development and validation of a predictive model for chemotherapy-associated thrombosis. Blood. 2008; 111: 4902-4907.

9. Palumbo A, Rajkumar SV, Dimopoulos MA, Richardson PG, San Miguel J, Barlogie $\mathrm{B}$, et al. Prevention of thalidomide- and lenalidomide-associated thrombosis in myeloma. Leukemia. 2008; 22: 414-423.

10. Zamagni E, Valdrè L, Palareti G, Cavo M. Prevention of VTE in multiple myeloma patients. Thrombosis Research. 2007; 120: S133-S136.

11. Palumbo A, Palladino $C$. Venous and arterial thrombotic risks with thalidomide: evidence and practical guidance. Ther Adv Drug Saf. 2012; 3: 255-266.

12. Kristinsson SY. Thrombosis in Multiple Myeloma. Hematology Am Soc Hematol Educ Program. 2010; 437-444.
Ann Hematol Oncol - Volume 4 Issue 2 - 2017

ISSN : 2375-7965 | www.austinpublishing group.com

Alves et al. (C) All rights are reserved
Citation: Alves C, Ramalheira S, Tavares M, Simas A, Rosales M, Milheiro MJ, et al. Thromboembolic Events in Multiple Myeloma Patients- Incidence in 235 Patients. Ann Hematol Oncol. 2017; 4(2): 1134. 